\title{
Validity of Simple Health Assessments to Evaluate Locomotive and Cognitive Functions of Late-Stage Older Adults in Japan
}

\author{
Eri Sarashina ${ }^{1}$, Katsuyoshi Mizukami ${ }^{1}$, Hanatsu Nagano $^{2}$ and Rezaul K Begg ${ }^{2}$ \\ ${ }^{1}$ Faculty of Health and Sport Sciences, University of Tsukuba, Japan \\ ${ }^{2}$ Institute of Sport, Exercise and Active Living (ISEAL), Victoria University, Australia
}

*Corresponding author: Katsuyoshi Mizukami, Faculty of Health and Sport Sciences, Graduate School of Comprehensive Human Sciences, University of Tsukuba, Tokyo, Japan, Tel: +81-3-39426453, E-mail: mizukami.katsuyos.ga@u.tsukuba.ac.jp

\begin{abstract}
Background: Due to the trend of ageing commonly seen in developed countries, late-stage older adults are expected to enhance their healthy life expectancy for sustainable healthcare systems. Sound locomotive and cognitive functions are the two important factors for independent lifestyles. While various institutions offer simple health assessments to check their health status, their validity still has room for debate. The current research, therefore, inspected whether simple health assessments would adequately reflect locomotive and cognitive functions.

Methods: Late-stage frail older adults $(n=19 ; 84.1 \pm 4.6 \mathrm{yrs}$; height $1.46 \pm 0.08 \mathrm{~m}$; body mass $49.6 \pm 8.6 \mathrm{~kg}$ ) recruited from the local aged-care facility undertook various examinations. Simple assessments included grip strength; single leg standing and sit-and-reach. Optitrack motion capture system recorded posture and treadmill gait at $100 \mathrm{~Hz}$. Locomotive functions were determined by step length $\&$ width, double support time and the indicator of tripping risk, Minimum Foot Clearance (MFC). Cognitive assessments were Mini-Mental State Examination (MMSE) and Trail Making Test-A (TMT-A). Pearson's correlation analysis examined interlinks between measured variables.

Results: Correlation results found that sit-and-reach and MFC were correlated. Greater grip strength was associated with symmetrical MFC control. Sit-and-reach correlated with MMSE scores. MMSE was negatively correlated with step width. TMT-A showed correlation with double support time.

Conclusions: Results of correlation analysis suggested that sit-and-reach and grip strength may predict optimum MFC control and associated tripping risks, while decreased cognitive functions may trigger cautious gait adaptations. Further research is required with a larger sample size for conclusive evidence.
\end{abstract}

\section{Keywords}

Gait impairment, Cognitive impairment, Simple health assessments, Falls prevention

\section{Introduction}

Ageing is the common tendency in developed countries, predicted to further escalate in the coming decades. This tendency is most profound in Japan, where $33 \%$ of the population was over 60-years-old [1]. Due to this increased trend of ageing, many age-associated health problems have arisen. In Japan, for example, annual tax revenue almost equalled medical costs in 2014 [2], therefore necessary to enhance healthy life expectancy of the senior population to sustain the social security system. For this reason, it is important for late-stage older adults (over $75 \mathrm{yrs}$ ) to slow down or possibly reverse the progress of frailty to reduce dependency on healthcare systems.

Two major obstacles for older adults to maintain independent lifestyles are loss of locomotive and cognitive functions. Loss of locomotive functions can be largely attributable to falls-related injuries. Yoshida and Kim reported that $20 \%$ of Japanese older adults experience a fall at least once a year [3]. As falls often cause severe injuries, falls prevention is important for older adults to sustain locomotive functions and active independent lifestyles. Cognitive impairment also hinders older adults from a social life. In 2014, 44 million people were suffering from dementia worldwide, predicted to steeply increase up to 65.7 million in 2030 [4]. In addition, $10-20 \%$ of older adults 
show symptoms of Mild Cognitive Impairment (MCl), the preceding stage of dementia [5].

For early detection of impairments and monitoring the health, regular health-checks should be encouraged for older individuals. For this purpose, 'basic' health-checks including grip strength, single leg standing and sit-andreach are often offered by health centres. Such simple assessments can help senior individuals to become aware of their own health due to accessibility within their own local community without incurring large costs. Nevertheless, questions still remain about the validity of these simple assessments. For example, grip strength is measured to assess falls risks based on the theory that it correlates with lower limb strength [6]. Considering previous gait studies on falls risks, assessment of upper limb extremity to predict falls during locomotion may need further study.

When considering age-associated gait changes and increased falls risks, head position was found to play a crucial role [7]. Forward tilted head has been characterised as one of the age-related negative postural characteristics with the deformed spine (e.g. Kyphosis). Spinal alignment could affect head movement during walking, and therefore, may correlate with locomotive functions. If interlinks were found between postural alignment and gait functions, results may suggest that correction of postural alignment could improve locomotive performance.

The current research thus aims to investigate the links between simple health-checks and the detailed assessments of locomotive and cognitive functions. Locomotive functions are described by spatio-temporal gait parameters and the tripping indicator, Minimum Foot Clearance (MFC) [8]. As tripping is the leading cause of falls [9], it will be valuable to examine whether simple assessments can predict tripping risks. Cognitive impairment was also reported to elevate falls risks possibly due to some areas in the brain governing simultaneously motor and cognitive functions (e.g. prefrontal lobe, basal ganglia and cerebellum) [10]. Further objective of the current research was, therefore, to explore interlinks between locomotive and cognitive functions.

\section{Methods}

\section{Participants}

Late-stage frail older adults ( $N=19 ; 84.1 \pm 4.6$ yrs.; Height $1.46 \pm 0.08 \mathrm{~m}$; Body mass $49.6 \pm 8.6 \mathrm{~kg}$ ) were recruited from the local aged-care facility in Japan to participate into the study. All participants possessed care-need certification $(2.9 \pm 1.3 ; 7$ point scale), which indicates eligibility to the Japanese government's financial support for their healthcare issues. Participants were classified as having both locomotor and cognitive impairments based on 'fundamental check-list' issued by Ministry of Health, Labour and Welfare of Japan [11]. Fundamental check-list is the standardised assessment for frailty status in Japan, while problems remain in the use of relatively generic questionnaire by non-specialist government officials [11]. Prior to commencement of the study, all participants signed informed consent, approved by the institutional ethics committee of University of Tsukuba.

\section{Protocol}

The participants undertook various examinations including simple assessments; locomotive functions; cognitive functions and posture. 3D motion capture system (Optitrack, Natural Point) recorded postural and locomotive data at $100 \mathrm{~Hz}$ and low-pass Butterworth filtered $(6 \mathrm{~Hz})$ to eliminate noise prior to analysis.

\section{Simple assessments}

Basic physical health was measured by grip strength, single leg standing and sit-and-reach. Grip strength was measured by Smedley-type Hand Dynamometer (Grip-D, TKK5401) and measured twice for both hands and the higher value was selected for analysis. Single leg standing was also recorded for both right and left stance ( 2 trials each). The best (longest) score was kept for analysis. Single leg standing was measured up to the maximum of 30 seconds. Sit-and-reach followed the conventional method to assess hamstring and lower back flexibility [12].

\section{Locomotive functions}

Gait testing was conducted on the treadmill at 0.3 $\mathrm{km} / \mathrm{h}$ to collect a minimum of 30 step cycles for analysis. Reflective markers were attached to the toe (i.e. superior surface of the distal end of the foot) and heel (i.e. proximal end of the foot) to model foot movement [13]. Heel contact and toe-off were determined by kinematic definitions [14]. Step length and width were defined as anterior-posterior and medio-lateral displacements between heels at heel contact (Figure 1). Double support time was the temporal period when both feet are in contact with the walking surface (i.e. from heel contact to contralateral toe-off).

As illustrated in Figure 1, Minimum Foot Clearance (MFC) was the vertical displacement of the toe from the walking surface during the mid-swing phase (7). Spatio-temporal parameters and MFC were described by mean \pm Standard Deviation (SD). Symmetry Index (SI) computed the degree of asymmetry for both spatio-temporal parameters and MFC [13].

$$
S I=\frac{(R-L)}{0.5 \times(R+L)} \times 100 \%
$$

Where $\mathrm{R} / \mathrm{L}$ indicating right/left foot leading parameter (Figure 1).

\section{Cognitive functions}

Both Mini-Mental State Examination (MMSE) [15] and Trail-Making Test-A (TMT-A) [16] tested partici- 


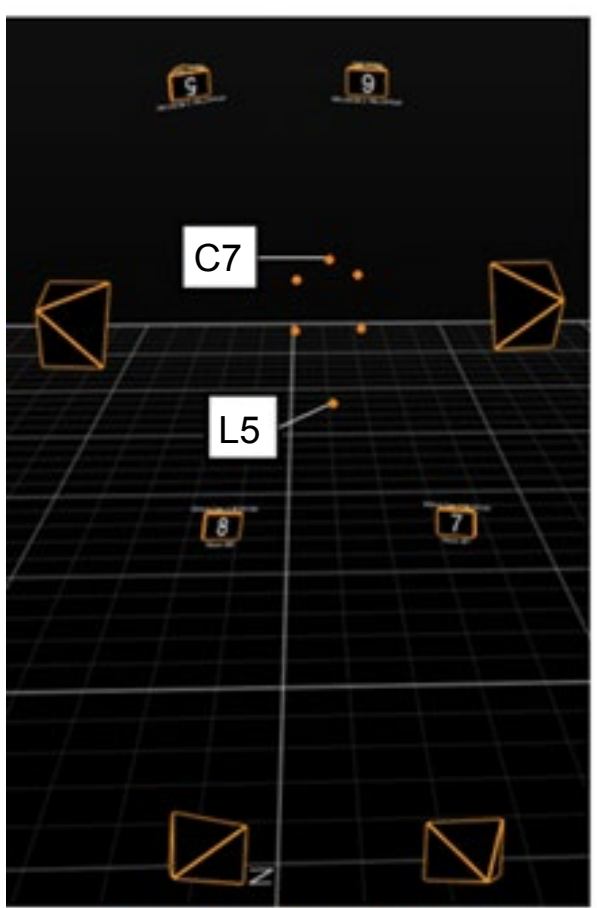

Marker \& camera setup

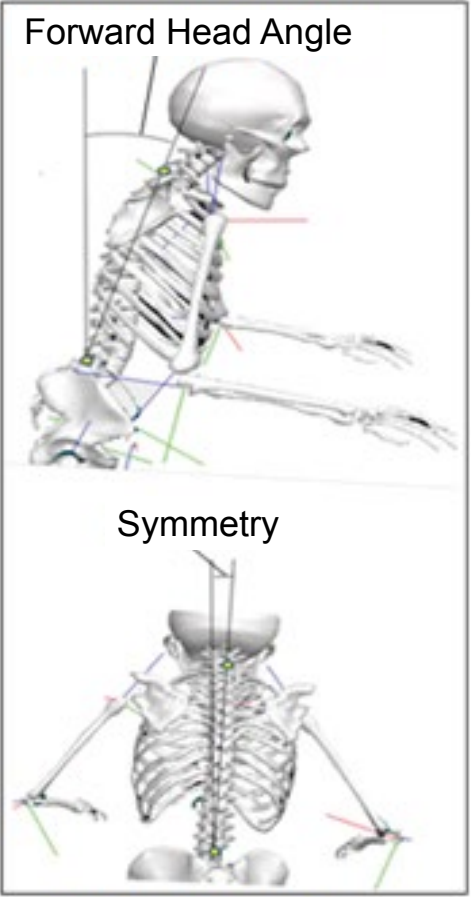

Posture definition

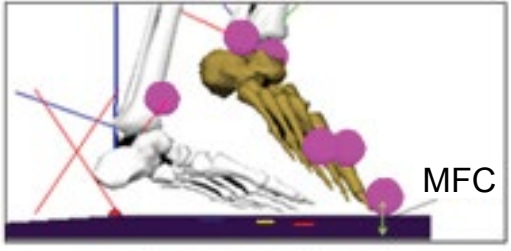

Definition of MFC

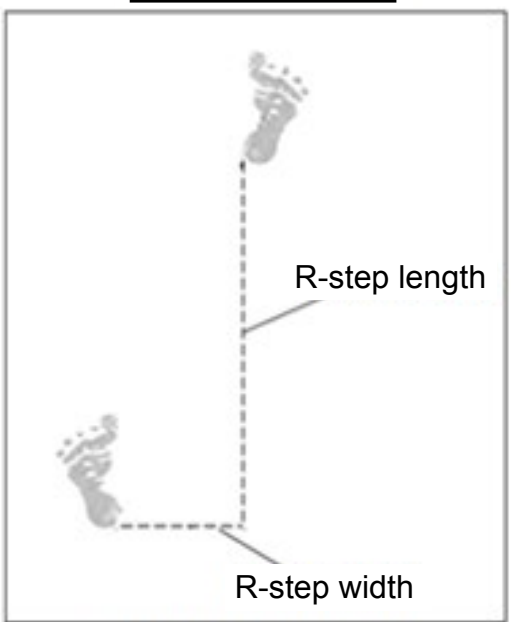

Step length \& width

Figure 1: (Left) Camera setup and posture analysis; (Middle) Definitions of posture analysis; (Right) Definitions of MFC and step length \& width.

pants' cognitive functions. MMSE evaluates the temporal and spatial orientation, memory skills, attention, oral and written language, and constructional abilities in two dimensions [15]. TMT-A examines executive function, in which a participant was asked to complete a task of connecting numbers from 1 to 25 and time taken to complete the task was measured [16]. In the current study, TMT-A was conducted while sitting in a chair and taking a concurrent task of constant foot-stepping (i.e. stepping and connecting numbers).

\section{Posture measurement}

Posture was determined by spinal alignment. Reflective markers were attached to C7 and L5. Angles were obtained in both sagittal and frontal planes to indicate forward head position and symmetrical alignment, respectively (Figure 1). Participants were asked to stand naturally and stationary for 5 seconds and average 3D locations of the two markers defined the backbone angles. Positive $(>0)$ angles indicated more forward head posture and asymmetrical spinal alignment [17].

\section{Correlations analysis}

Pearson's correlations analysis was performed to inspect interlinks between dependent variables (i.e. simple assessments, cognitive/locomotive functions, posture). Significant correlations were determined when computed $p$-values were less than 0.05 .

\section{Results}

Table 1 summarises the group data. Table 2 describes the correlation patterns between simple assessments and i) Locomotive functions; ii) Cognitive functions and iii) Posture.

Other notable correlations were as follows. MMSE and TMT-A showed negative correlations $(r=-0.508, p<$ 0.05 ), indicating that increased MMSE scores were associated with shorter TMT-A. For interlinks between cognitive and locomotive functions, TMT-A and step width were positively correlated $(r=0.715, p<0.01)$. MMSE was negatively correlated with double support time ( $r$ $=-0.536, p<0.05)$. Trends were seen between MFC and MMSE scores $(r=465, p=0.052)$; and shorter TMT-A ( $r$ $=-0.437, p=0.070)$. Interestingly, mean and SD of MFC were correlated $(r=0.704, p<0.01)$.

Posture was not correlated with simple assessments in Table 2. Both forward head position and spinal symmetry were, however, highly correlated with each other $(r=0.744, p<0.01)$. Longer double support time was associated with both greater forward head position ( $r$ $=0.665, p<0.01)$ and less symmetrical backbone alignment $(r=0.529, p<0.05)$.

\section{Discussion}

\section{Validity of simple assessments}

The current research examined whether simple health assessments would validly reflect health-statuses of latestage older adults. First, the previous research reported the link between grip strength and falls risks because individuals with sufficient grip strength tend to also demonstrated adequate lower limb strength [6]. The current research found correlations between grip strength and MFC 
Table 1: Group data $(\mathrm{n}=19)$.

\begin{tabular}{|c|c|c|c|}
\hline \multicolumn{2}{|l|}{ Parameters } & \multirow{2}{*}{$\begin{array}{l}\text { Unit } \\
\text { yrs }\end{array}$} & \multirow{2}{*}{$\begin{array}{l}\text { Mean } \pm \text { inter-subject variability } \\
84.1 \pm 4.6\end{array}$} \\
\hline Anthropometric/Educational data & Age & & \\
\hline & Height & $\mathrm{m}$ & $1.46 \pm 0.08$ \\
\hline & Body mass & $\mathrm{kg}$ & $49.6 \pm 8.6$ \\
\hline & BMI & Unitless & $23.2 \pm 3.5$ \\
\hline & Education & yrs. & $9.5 \pm 2.8$ \\
\hline \multirow[t]{5}{*}{ Simple assessments } & Grip (right) & $\mathrm{kg}$ & $17.0 \pm 3.6$ \\
\hline & Grip (left) & $\mathrm{kg}$ & $15.9 \pm 3.1$ \\
\hline & Single leg standing (right) & s & $12.8 \pm 29.1$ \\
\hline & Single leg standing (left) & s & $11.3 \pm 24.1$ \\
\hline & Sit-and-reach & $\mathrm{cm}$ & $27.6 \pm 9.9$ \\
\hline \multirow[t]{2}{*}{ Cognitive functions } & MMSE & Unitess & $23.7 \pm 4.1$ \\
\hline & TMT-A (step) & $\mathrm{s}$ & $271.8 \pm 156.3$ \\
\hline \multirow[t]{2}{*}{ Posture } & Head forward angle & $\circ$ & $12.4 \pm 12.4$ \\
\hline & Back symmetry & $\circ$ & $4.4 \pm 4.8$ \\
\hline \multirow[t]{12}{*}{ Locomotive functions } & Step length (mean) & $\mathrm{m}$ & $0.08 \pm 0.03$ \\
\hline & Step length (SD) & $\mathrm{m}$ & $0.03 \pm 0.01$ \\
\hline & Step length (SI) & $\%$ & $13.3 \pm 62.2$ \\
\hline & Step width (mean) & $\mathrm{m}$ & $0.10 \pm 0.02$ \\
\hline & Step width (SD) & $\mathrm{m}$ & $0.01 \pm 0.01$ \\
\hline & Step width (SI) & $\%$ & $0.12 \pm 3.74$ \\
\hline & Double support time (mean) & $\mathrm{s}$ & $0.25 \pm 0.13$ \\
\hline & Double support time (SD) & s & $0.12 \pm 0.08$ \\
\hline & Double support time (SI) & $\%$ & $-13.0 \pm 37.5$ \\
\hline & MFC (mean) & $\mathrm{cm}$ & $3.86 \pm 0.88$ \\
\hline & MFC (SD) & $\mathrm{cm}$ & $0.94 \pm 0.64$ \\
\hline & MFC (SI) & $\%$ & $19.59 \pm 29.19$ \\
\hline
\end{tabular}

Table 2: Correlations between basic data and cognitive function, posture, step length \& width and MFC.

\begin{tabular}{|c|c|c|c|c|c|c|}
\hline & & \multicolumn{5}{|c|}{ Simple assessments } \\
\hline & & R-Grip & L-Grip & R-SLS & L-SLS & Sit-\&-reach \\
\hline \multirow[t]{9}{*}{ Locomotive } & Step length (mean) & 0.004 & -0.036 & -0.101 & -0.066 & 0.035 \\
\hline & Step length (SI) & 0.410 & 0.338 & -0.047 & -0.006 & -0.035 \\
\hline & Step width (mean) & -0.107 & -0.278 & -0.0046 & -0.015 & -0.093 \\
\hline & Step width (SI) & -0.250 & -0.431 & 0.171 & 0.216 & 0.400 \\
\hline & DST (mean) & -0.109 & -0.201 & -0.042 & 0.023 & -0.306 \\
\hline & DST (SI) & -0.313 & 0.281 & 0.031 & 0.049 & -0.321 \\
\hline & MFC (mean) & -0.069 & -0.087 & 0.395 & 0.345 & $0.623^{* *}$ \\
\hline & MFC (SD) & -0.013 & 0.203 & 0.123 & 0.051 & $0.509^{*}$ \\
\hline & $\mathrm{MFC}(\mathrm{SI})$ & -0.462 & $-0.685^{*}$ & 0.257 & 0.294 & 0.049 \\
\hline \multirow[t]{2}{*}{ Cognitive } & MMSE & 0.072 & 0.021 & 0.410 & 0.451 & $0.666^{* *}$ \\
\hline & TMT-A & 0.009 & -0.083 & -0.373 & -0.411 & -0.321 \\
\hline \multirow[t]{2}{*}{ Posture } & Head forward angle & 0.070 & 0.102 & -0.209 & -0.191 & -0.376 \\
\hline & Back symmetry & -0.338 & -0.308 & -0.175 & -0.150 & -0.316 \\
\hline
\end{tabular}

Note: DST: Double Support Time; MFC: Minimum Foot Clearance; MMSE: Mini-Mental State Examination; TMT-A: Trail Making Test-A; SD: Standard Deviation; SI: Symmetry Index.

Bold indicating $p<0.1$; *indicating $p<0.05$; * indicating $p<0.01$.

symmetry. Individuals with greater grip strength may, therefore, demonstrate more symmetrical MFC, possibly indicating reduced tripping risks [14]. Symmetrical MFC control demands fine-endpoint control rather than gross muscle activity. Martin, et al. reported the association between grip strength and hand dexterity [18]. Grip strength may be, therefore, associated with lower limb strength, controlling dexterity tasks of the distal end of the lower $\operatorname{limb}$ (i.e. toe).

Strong positive correlations between MFC and sit-andreach implied that knee/hip motions related to flexibili- ty of hamstrings and lower back may increase swing toe clearance and reduce tripping risks, since these joint motions control MFC [19]. In contrast, correlations between sit-and-reach and MFC variability (SD) suggest that flexibility could cause more variable MFC control, therefore disadvantageous for tripping prevention [8]. This correlation may be, however, secondary to positive correlations between mean and SD of MFC ( $r=0.704)$. Higher swing foot clearance can be achieved by a combination of multiple joint motions and therefore, it may be difficult to retain low MFC variability while increasing mean MFC. 
Despite no significance, the trend $(p<0.1)$ was found between single leg standing and cognitive functions. The certain brain parts such as cerebellum may be responsible for both balance and cognitive functions [9]. Similar results were obtained between sit-and-reach and cognitive functions (i.e. MMSE), which is consistent with the previous report [20]. With increased sample size, significant correlations may be validated between cognitive functions and flexibility. These implications indicate the possibilities that (i) Impaired executive function could cause inflexibility or/and (ii) Flexibility training may improve cognitive functions.

\section{Interlinks between locomotive and cognitive functions}

The research also inspected associations between locomotive and cognitive functions. The current research revealed the trend of association between declined cognitive functions and lower MFC. Correlation patterns were identified between higher TMT-A and larger step width; and between increased double support time and lower MMSE scores. Increased step width and double support time are typical gait adaptations to secure gait [7]. Cognitive impairment was, therefore, considered to trigger cautious gait adaptations to secure dynamic balance.

\section{Posture}

Postural data were not correlated with simple assessments and cognitive functions. With locomotive functions, suboptimal posture seems to require longer double support time possibly due to difficulty in maintaining dynamic balance.

\section{Limitations in the research}

The current study was unique in evaluating the validity of conventional health checks for late-stage older adults. On average, the group had $\mathrm{MCl}$ shown in average MMSE scores of 23.7 [21]. Mostly due to treadmill walking velocity of $0.3 \mathrm{~km} / \mathrm{s}$, step length was remarkably short. In such slow belt speed, normal stride is difficult to take. This experimental protocol was adopted because many of the participants had severe locomotive impairment, where walking without assisting device was challenging for some participants. Since even healthy older adults could not walk properly on the treadmil [22], overground gait testing may be the alternative option for this population. In contrast to our expectation, significant correlations between postural alignment and locomotive parameters were not identified in this study. More detailed postural assessment may, however, potentially reveal association between posture and gait functions. For future research, a larger sample size should be also tested for the conclusive evidence.

\section{Future directions}

In the most aged country, Japan, non-specialist assessors tend to evaluate locomotive and cognitive func- tions of late-stage older adults rather subjectively due to the lack of established standards. A greater variety of valid simple assessments should be devised to reliably and quantitatively evaluate senior adults' health statuses. Use of such simple assessments can be standardised to determine the level of reliance on governmental health-support. Hints for effective preparation for further ageing in future can be possibly found from the case study of Japan.

\section{Acknowledgments}

The study was supported by Shinagawa Award of Tokyo for recruitment of the participants.

\section{References}

1. United Nations Department of Economic and Social Affairs (2015) Population Division. World Population Ageing 138.

2. Ministry of Health, Labour and Welfare (2014) 2014 Edition: Annual Health, Labour and Welfare Reportfor the Realization of a Society of Health and Longevity.

3. Yoshida H, Kim H (2006) [Frequency of falls and their prevention]. Clin Calcium 16: 1444-1450.

4. Kálmán J, Juhász $A$, Rimanóczy $A$, Palotás $A$, Palotás $M$, et al. (2003) The nitric oxide synthase-3 codon 298 polymorphism is not associated with late-onset sporadic Alzheimer's dementia and Lewy body disease in a sample from Hungary. Psychiatr Genet 13: 201-204.

5. Petersen RC (2011) Clinical Practice. Mild Cognitive Impairment. N Engl J Med 364: 2227-2234.

6. Wahba H, Abdul-Rahman S, Mortagy A (2013) Handgrip strength and falls in community-dwelling Egyptian seniors. Adv Aging Res 2: 109-114.

7. Muir SW, Gopaul K, Montero Odasso MM (2012) The role of cognitive impairment in fall risk among older adults: a systematic review and meta-analysis. Age Ageing 41: 299308.

8. Winter DA (1995) Human balance and posture control during standing and walking. Gait Posture 3: 193-214.

9. Begg R, Best R, Dell'Oro L, Taylor S (2007) Minimum foot clearance during walking: strategies for the minimisation of trip-related falls. Gait Posture 25: 191-198.

10. Blake AJ, Morgan K, Bendall MJ, Dallosso H, Ebrahim SB, et al. (1988) Falls by elderly people at home: prevalence and associated factors. Age Ageing 17: 365-372.

11. Satake S, Senda K, Hong YJ, Miura H, Endo $H$, et al. (2016) Validity of the Kihon Checklist for assessing frailty status. Geriatr Gerontol Int 16: 709-715.

12. Mayorga-Vega D, Merino-Marban R, Viciana J (2014) Criterion-Related Validity of Sit-and-Reach Tests for Estimating Hamstring and Lumbar Extensibility: a Meta-Analysis. J Sports Sci Med 13: 1-14.

13. Nagano H, Begg RK, Sparrow WA, Taylor S (2011) Ageing and limb dominance effects on foot-ground clearance during treadmill and overground walking. Clin Biomech (Bristol, Avon) 26: 962-968.

14. O'Connor CM, Thorpe SK, O'Malley MJ, Vaughan CL (2007) Automatic detection of gait events using kinematic data. Gait Posture 25: 469-474.

15. Folstein MF, Folstein SE, McHugh PR (1975) Mini-Mental 
State. A practical method for grading the cognitive state of patients for the clinician. J Psychiat Res 12: 189-198.

16. Sanchez-Cubillo I, Perianez JA, Adrover-rong D, Rodríguez-Sánchez JM, Ríos-Lago M, et al. (2009) Construct validity of the trail making test: Role of task-switching, working memory, inhibition/interference control, and visuomotor abilities. J Int Neuropsychol Soc 15: 438-450.

17. Benedetti MG, Berti L, Presti C, Frizziero A, Giannini S (2008) Effects of an adapted physical activity program in group of elderly subjects with flexed posture: clinical and instrumental assessment. J Neuroeng Rehabil 5: 32.

18. Martin JA, Ramsay J, Hughes C, Peters DM, Edwards MG (2015) Age and grip strength predict hand dexterity in adults. PLoS One 10: e0117598.
19. Moosabhoy MA, Gard SA (2006) Methodology for determining the sensitivity of swing leg toe clearance and leg length to swing leg joint angles during gait. Gait Posture 24: 493-501.

20. Taylor-Piliae RE, Newell KA, Cherin R, Lee MJ, King AC, et al. (2010) Effects of Tai Chi and Western exercise on physical and cognitive functioning in healthy community-dwelling older adults. J Aging Phys Act 18: 261-279.

21. Trzepacz PT, Hochstetler H, Wang S, Walker B, Saykin AJ (2015) Relationship between the Montreal Cognitive Assessment and Mini-mental State Examination for assessment of mild cognitive impairment in older adults. BMC Geriatr 15: 107.

22. Wass E, Taylor NF, Matsas A (2005) Familiarisation to treadmill walking in unimpaired older people. Gait Posture 21: $72-79$. 